\title{
Umgang mit Gewalt besser und mehr schulen
}

Kompetenz und Empathie stärken Gewalt in der Pflege wird in Praxis und Ausbildung noch viel zu wenig Raum gegeben. Abhilfe schaffen soll umfangreiches Schulungsmaterial, herausgegeben vom „Zentrum für Qualität in der Pflege“. Pflegeexpertinnen und -experten definieren Gewalt, geben Tipps und fördern anhand von Fallbeispielen Diskussionen über adäquates Verhalten in der Pflege.

$\mathbf{G}$ ewalt sowohl in der häuslichen als auch in der ambulanten und stationären Pflege ist keine Seltenheit. Da sind sich die Expertinnen und Experten weitgehend einig. Belastbares Datenmaterial gibt es trotzdem kaum. Die Dunkelziffer ist hoch. Mit ein Grund dafür ist die Tatsache, dass Gewalt ganz unterschiedlich definiert und ebenso unterschiedlich wahrgenommen wird. „Gewalt ist eben so viel mehr als nur strafrechtlich relevante, zur Anzeige gebrachte und damit statistisch auswertbare Übergriffe“, unterstreicht Daniela Sulmann, Pflegeexpertin und Bereichsleiterin Projekte im „Zentrum für Qualität in der Pflege“ (ZQP) in Berlin. Missachtung, Ignoranz, Vernachlässigung und Übergriffigkeit: Auch das gilt als Gewalt an Schutzbefohlenen und diese Gewalt kann sowohl von den Pflegenden als auch von zu pflegenden Menschen ausgehen. Hinzukommt die Mentalität vieler alter und

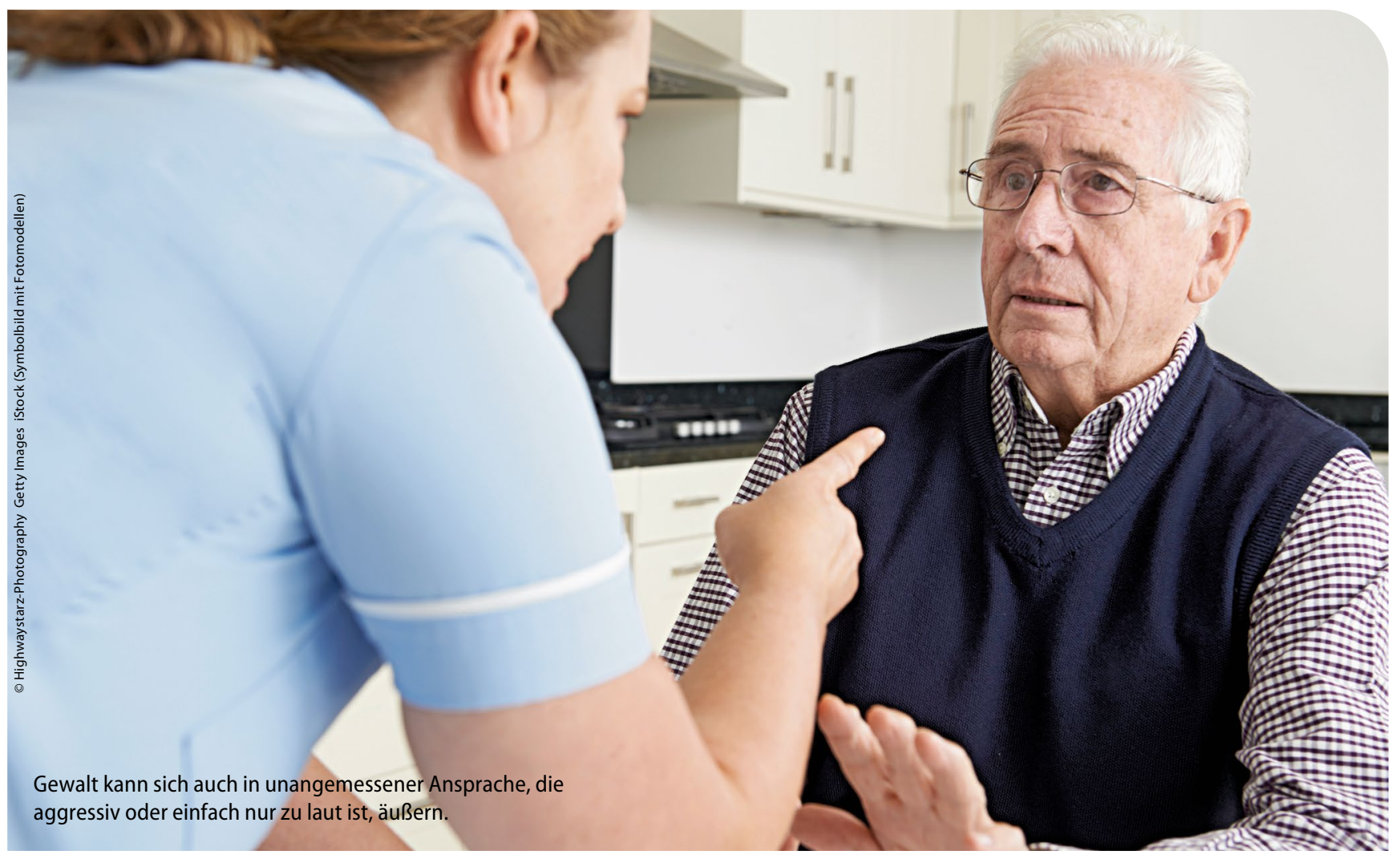




\section{PFLEGE PERSPEKTIVEN}

sehr alter Menschen. Daniela Sulmann: „Die derzeit zu pflegenden hochaltrigen Menschen gehören einer Generation an, die teilweise vielleicht bescheidener ist als nachfolgende Generationen, weniger hohe Erwartungen an Dienstleistungen hat und nicht zur Last fallen will. Sie sind oft auch gar nicht in der Verfassung, Missstände zu beklagen. Das wird sich möglicherweise ändern - wenn die jüngeren Generationen, deren Position als Verbraucherinnen und Verbraucher deutlich gestärkt wurde, pflegebedürftig werden.“

Mit ein Grund, warum die Krankenschwester und Dipl. Pflegewirtin es für wichtig erachtet, jetzt die Weichen dafür zu stellen, dass das Pflegepersonal und der Pflege-Nachwuchs für das Thema "Gewalt in der Pflege" sensibilisiert wird und entsprechende Strategien an die Hand bekommt, mit denen sich Gewalt auf beiden Seiten verhindern lässt. Helfen soll dabei das umfangreiche Schulungsmaterial, welches das ZQP in den vergangenen Jahren entwickelt und veröffentlicht hat.

\section{Überforderung ist nur eine Komponente}

Im Vorfeld initiierte das ZQP in Zusammenarbeit mit der Deutschen Hochschule der Polizei eine Erhebung, deren Ergebnisse 2020 veröffentlicht wurden. Mitarbeitende in der Pflege antworteten auf die Frage, welche Formen der Gewalt sie in den vergangenen vier Wochen beobachtet hatten. $69 \%$ der Befragten berichteten von verbaler Gewalt, 33\% von körperlicher Gewalt und 10\% sogar von sexuellen Übergriffen. Wer die Gründe für dieses inakzeptable Verhalten nur in knappen Zeitbudgets und einer zu dünnen Personaldecke sucht, macht es sich nach Ansicht der Expertin zu leicht. „Überforderung des Personals ist nur eine Komponente eines sehr komplexen Problems. Mangelndes Fachwissen, eine eingeschränkte soziale Kompetenz und individuelle Komponenten spielen ebenfalls eine nicht zu unterschätzende Rolle. Pflegerinnen und Pfleger etwa, die selbst Gewalt erlebt haben, von Haus aus eher nervös und angespannt sind und dazu vielleicht weniger ausgeprägte Fachkompetenzen haben, sind eher gefährdet, aggressiv und gewalttätig zu handeln. Da können gezielte Schulungsmaßnahmen vielfach vorbeugen“, erklärt Daniela Sulmann.

\section{Rollentausch, um Bedürfnisse nachzuvollziehen}

Möglich wird dies unter anderem mit der Methode "Schattentage“, die ebenfalls im ZQP entwickelt wurde. Für zwei bis drei Tage tauschen Pflegerinnen oder Pfleger die Rollen mit ihren Pflegebefohlenen. Oder sie begleiten einen Pflegebedürftigen als „Schatten“ und beobachten, was passiert. Mit diesem innovativen Ansatz sollen Pflegekräfte befähigt werden, die Bedürfnisse und Wünsche von pflegebedürftigen Menschen besser nachzuvollziehen und diese dann auch entsprechend im Berufsalltag zu berücksichtigen. Die Expertin ist sicher: „Erst bei einem solchem Rollenwechsel erleben Pflegerinnen und Pfleger, was es heißt, bis in die intimsten Momente hinein abhängig von anderen zu sein. Die Erfahrungen zeigen, dass schon nach ganz kurzer Zeit die Pflegekräfte die Situation als schwer bis unerträglich erleben."

\section{Alles, was Schaden oder Leid zufügt}

Beteiligt an der Erarbeitung des Schulungsmaterials waren Pflegewissenschaftler und -wissenschaftlerinnen sowie Mitarbeitende in Pflegeeinrichtungen, ambulanten Diensten und Pflegeschulen. In

\section{EIN FALLBEISPIEL ZUM THEMA AGgRESSIONEN:}

Frau W. lebt seit dem Tod ihres Mannes bei ihrer Tochter und ihrem Schwiegersohn. Sie kann sich nicht mehr gut bewegen und ist auf Hilfe angewiesen. Der Pflegedienst wurde erst vor kurzem für die häusliche Krankenpflege beauftragt.

Perspektive 1 aus der Sicht der Pflegebedürftigen: „Vor einiger Zeit bin ich zu meiner Tochter und meinem Schwiegersohn gezogen. Mein Haushalt wurde aufgelöst und ich wohne jetzt im ehemaligen Kinderzimmer meiner Enkelin. Die meiste Zeit verbringe ich hier drin. Ich muss dankbar sein, dass sie sich um mich kümmern. Ich habe nur noch sie. Allein komme ich mit dem Rollator nicht aus dem Haus. Und Besuch bekomme ich ja auch nicht, weil ich hier niemanden kenne. Aber es ist nett, dass Herr G. nun regelmäßig vorbeikommt. Er ist immer so behutsam und sehr geduldig mit mir."

Perspektive 2 aus der Sicht des Pflegers: „Ich komme seit ein paar Wochen zu Frau W., um den Ulcus Cruris zu behandeln. Als es neulich so heiß war, hatte Frau W. ausnahmsweise ein kurzärmeliges T-Shirt an. Da habe ich eigenartige Druckstellen an ihren Armen bemerkt, die wie Fingerabdrücke aussahen. Als sich sie auf die Stellen ansprach, meinte sie, sie stoße sich regelmäßig überall. Das kann natürlich stimmen. Das Zimmer ist durch die Möbel der Enkelin nicht gerade für pflegebedürftige Menschen geeignet. Aber irgendwie habe ich ein ungutes
Gefühl. Sie wirkt auch mich sehr scheu und übertrieben respektvoll, Ich habe schon mal versucht, sie dezent dazu zu befragen. Aber Frau S. lässt uns praktisch nie allein. Sie kommt mir überfürsorglich vor und kontrolliert alles, was passiert. Also typische Anzeichen dafür, dass da etwas nicht stimmt. Den Kollegen ist beim Beratungsgespräch scheinbar nichts aufgefallen. Aber ich hatte ja auch in den ersten paar Tagen noch keinen Verdacht. Vielleicht war es ja auch nur ein Ausrutscher?"

Perspektive 3 aus Sicht der Tochter: „Meine Mutter wurde in den letzten Jahren zunehmend hilfsbedürftig. Allein kam sie in ihrer Wohnung nicht zurecht. Deswegen wohnt sie inzwischen bei uns. Wir hatten nicht immer das beste Verhältnis, aber als Familie muss man eben zusammenhalten. Geld gibt es von der Pflegekasse ja auch. Bei uns hat sie ihr eigenes Zimmer und alles, was sie braucht. Wir versuchen, es ihr recht zu machen - aber sie macht es uns nicht immer leicht. Oft habe ich den Eindruck, sie lässt sich absichtlich hängen. Das ärgert mich dann sehr." 
dem mit Zeichnungen illustrierten Ratgeber „Gewalt vorbeugen Praxistipps für den Pflegealltag“ definiert das ZQP zunächst einmal allgemeingültig, was in der Pflege unter Gewalt zu verstehen ist: Alles was pflegebedürftigen Menschen „Schaden oder Leid zufügt - körperlich, seelisch oder finanziell“. Weiter geht es mit den zu beachtenden Grundregeln: die Rechte von Pflegebedürftigen kennen, ihre Selbstbestimmung beachten, ihre Selbstständigkeit und ihr Selbstvertrauen erhalten, Sicherheit geben, Zeitdruck vermeiden und für schöne Momente sorgen. Ergänzt werden die Ausführungen mit Praxistipps: Wie kann man

_ das Selbstwertgefühl von pflegebedürftigen Menschen unterstützen,

- das Verhalten der Seniorinnen und Senioren besser verstehen lernen,

- akute Aggressionen bereits im Ansatz entschärfen,

- freiheitsentziehende Maßnahmen vermeiden,

- mit dem eigenen Gewaltpotential umgehen lernen

- Wege finden, gut für sich selbst zu sorgen und

- Gefahrensituation besser einschätzen lernen?

Im Anhang finden sich zudem nützliche Adressen, die man sowohl als pflegender Angehöriger als auch als professionelle Pflegerin oder Pfleger immer zu Hand haben sollte. Dazu gehören Notfalltelefonnummern, Schulungsangebote, Kurse, nützliche Hilfsmittel sowie die Hinweise zu Entlastungsangeboten und Selbsthilfegruppen.

„Gewalt gegen pflegebedürftige Menschen verhindern - was man wissen sollte - und was man tun kann" erweitert den Ratgeber und benennt die Folgen von Gewalt gegenüber zu pflegenden Menschen: „Psychische Folgen sind zum Beispiel Stress, Unruhe, Angst, Verzweiflung, Schlafstörungen, Kraft- und Antriebslosigkeit, Gefühle der Ohnmacht oder Erniedrigung und Aggressionen.“ Die Anzeichen, die auf Gewalt hindeuten, werden zusammengestellt und Tipps gegeben, wie man die zu pflegenden Menschen vor Gewalt schützen kann:

- Anzeichen wahrnehmen und Beobachtungen ansprechen

- Hilfe anbieten, Beschwerden anbringen, dokumentieren

- Verantwortliches Personal verständigen und Rat holen

_ Ärztliche Untersuchung anregeund Polizei verständigen.

\section{Was geht? Was ist inakzeptabel?}

Für die praktische Arbeit mit dem Material können Plakate, Präsentationsfolien und Arbeitsblätter heruntergeladen werden. Tipps zur Vorbereitung, Durchführung und Nachbereitung erleichtern die Aufbereitung des Themas für Lehrende in der Pflege und Fallbeispiele erleichtern den Einstieg in die Diskussion.

Zielgruppen für die Arbeit mit dem Material sind sowohl Auszubildende in Pflegeberufen als auch das Personal von Pflegeeinrichtungen. Daniela Sulmann hat beobachtet, dass Gewalt in der Ausbildung derzeit eher rudimentär thematisiert wird und wenn, dann hauptsächlich in Verbindung mit an Demenz Erkrankten oder in der psychiatrischen Pflege. Sie hat erkannt: „Sowohl in den Schulungen als auch in der Einarbeitung neuer Pfleger und Pflegerinnen fehlt es mitunter an der Vermittlung klarer Wertmaßstäbe und Orientierungshilfen. Was geht und was geht nicht? Was wird akzeptiert und was ist inakzeptabel? Das wird auf der Leitungsebene nicht immer klar definiert, artikuliert und als Richtlinien vorgegeben. Außerdem ist es wichtig, deutlich zu machen, dass fach- liche Kompetenz nicht gleichbedeutend mit Sensibilität und Empathie ist und diese wiederum keine Garanten für fachliche Kompetenz sind. Beides ist aber wichtig und wir wollen mit unseren Materialien beides stärken: Fachwissen sowie Sensibilität und Empathie. Denn sowohl in der Praxis als auch in der Ausbildung sehen wir in diesem Bereich einen erheblichen Bedarf." Immerhin: Zwischen 50.000 bis 60.000 werden die Schulungsmaterialien pro Jahr angeklickt. Ein positives Zeichen dafür, dass das Thema „angekommen“ ist.

\section{Bisher nur Hinweise zu Pandemie-Auswirkungen}

Inwieweit die Corona-Pandemie tatsächlich noch einmal für eine Zunahme von Fällen körperlicher und verbaler Gewalt gegen Pflegebedürftige gesorgt hat, ist derzeit noch völlig offen. Die Expertin sieht allerdings Hinweise dafür, dass die Belastungen für professionell Pflegende in der Pandemie noch einmal größer geworden sind - mit entsprechenden Auswirkungen auf die Situation der zu pflegenden Menschen. „Es ist durchaus vorstellbar, dass Vernachlässigung und freiheitentziehende Maßnahmen in den vergangenen Monaten noch einmal häufiger aufgetreten sind als in der Vor-Corona-Zeit.“ Bei allem Verständnis für die schwierige Situation des Personals, Daniela Sulmann nimmt Pflegerinnen und Pfleger dennoch in die Pflicht. „Zeitdruck beziehungsweise Stress darf nicht als Rechtfertigung dafür dienen, Gewalt zu tolerieren. Pflegende müssen aktiv zum Schutz Pflegebedürftiger eingreifen, wenn deren Wohl gefährdet ist - das liegt in ihrer Verantwortung." Und sie fügt an: „Pfleger und Pflegerinnen sollten sich immer wieder bewusstmachen: Sie sind dem System und der Arbeitssituation nicht völlig ausgeliefert - aber pflegebedürftige Menschen sind in einer äußerst vulnerablen, abhängigen Lage. Die Tatsache, dass Pflegepersonal überall händeringend gesucht wird, sollte den Pflegenden Mut geben, Verbesserungen einzufordern - und im Zweifelsfall auch den Arbeitgeber zu wechseln.“

Annette Lübbers

\section{PFLEGE EINFACH MACHEN}

\author{
Es besteht erheblicher Nachholbedarf bei der Beschäftigung \\ mit dem Umgang und der Vermeidung von Gewalt in der \\ Aus- und Weiterbildung. \\ Das Zentrum für Qualität in der Pflege (ZQP) stellt umfangrei- \\ ches Schulungsmaterial zum Thema Gewaltprävention zu \\ Verfügung.
}

Schlüsselwörter: Gewaltprävention, Empathie, Kommunikation, Schulung

Links zu Materialien des ZQP zum Download finden Sie über das Heilberufe eMag auf springerpflege.de 